Provided for non-commercial research and education use. Not for reproduction, distribution or commercial use.

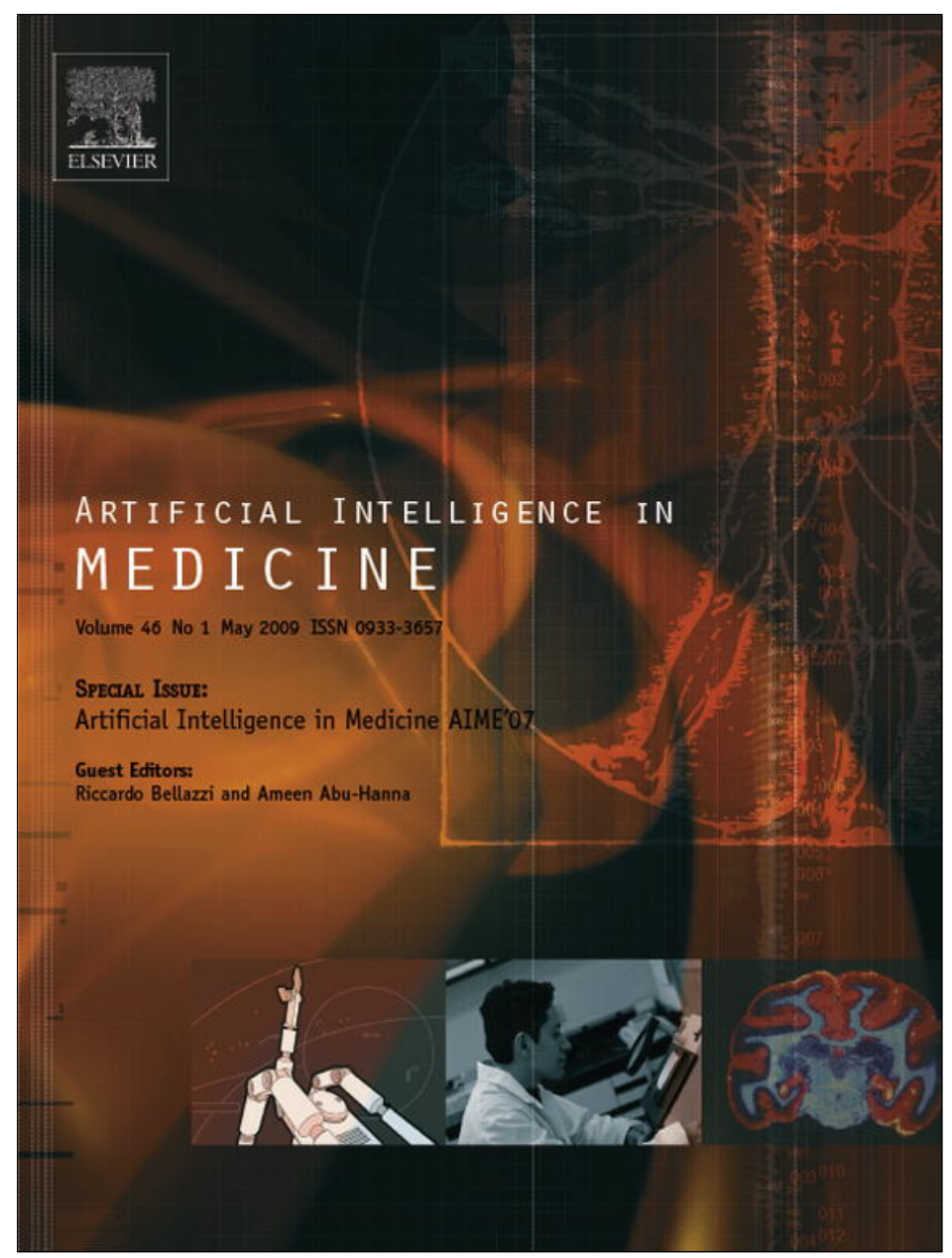

This article appeared in a journal published by Elsevier. The attached copy is furnished to the author for internal non-commercial research and education use, including for instruction at the authors institution and sharing with colleagues.

Other uses, including reproduction and distribution, or selling or licensing copies, or posting to personal, institutional or third party websites are prohibited.

In most cases authors are permitted to post their version of the article (e.g. in Word or Tex form) to their personal website or institutional repository. Authors requiring further information regarding Elsevier's archiving and manuscript policies are encouraged to visit:

http://www.elsevier.com/copyright 


\title{
Agentification of Markov model-based segmentation: Application to magnetic resonance brain scans
}

\author{
Benoit Scherrer $^{a, b, c}$, Michel Dojat ${ }^{a, c, *}$, Florence Forbes ${ }^{c, d}$, \\ Catherine Garbay ${ }^{\mathrm{b}, \mathrm{c}}$
}

${ }^{a}$ Grenoble Institut des Neurosciences (GIN), Centre de Recherche Institut national de la santé et de la recherche medicale (Inserm) U 836, E5 Chemin Fortuné Ferrini, La Tronche, BP 170, 38042 Grenoble Cedex 09, France

${ }^{\mathrm{b}}$ Centre National de la Recherche Scientifique (CNRS), Grenoble Informatics Laboratory (LIG), Batiment IMAG B, 385 avenue de la Bibliothèque, 38400 Saint Martin d'Hères, France

' Université Joseph Fourier, BP 53, 38041 Grenoble Cedex 9, France

'Laboratoire Jean Kuntzmann, MISTIS, ZIRST, 655 avenue de l'Europe, Montbonnot, 38334 Saint Ismier Cedex, France

Received 14 December 2007; received in revised form 22 August 2008; accepted 22 August 2008

\section{KEYWORDS}

Medical imaging;

Markov random field;

Multiagents system;

Distributed

expectation

maximization;

Magnetic resonance

brain scan

segmentation

\begin{abstract}
Summary
Objective: Markov random field (MRF) models have been traditionally applied to the task of robust-to-noise image segmentation. Most approaches estimate MRF parameters on the whole image via a global expectation-maximization (EM) procedure. The resulting estimated parameters are likely to be uncharacteristic of local image features. Instead, we propose to distribute a set of local MRF models within a multiagent framework.

Materials and methods: Local segmentation agents estimate local MRF models via local EM procedures and cooperate to ensure a global consistency of local models. We demonstrate different types of cooperations between agents that lead to additional levels of regularization compared to the standard label regularization provided by MRF. Embedding Markovian EM procedures into a multiagent paradigm shows interesting properties that are illustrated on magnetic resonance (MR) brain scan segmentation.
\end{abstract}

\footnotetext{
* Corresponding author at: Grenoble Institut des Neurosciences (GIN), Centre de Recherche Institut national de la santé et de la recherche medicale (Inserm) U 836, E5 Chemin Fortuné Ferrini, La Tronche, BP 170, 38042 Grenoble Cedex 09, France.

Tel.: +334565206 01; fax: +33456520598.

E-mail addresses: benoit.scherrer@imag.fr (B. Scherrer), michel.dojat@ujf-grenoble.fr (M. Dojat), florence.forbes@inrialpes.fr (F. Forbes), catherine.garbay@imag.fr (C. Garbay).
} 
Results: A cooperative tissue and subcortical structure segmentation approach is designed with such a framework, where both models mutually improve. Several experiments are reported and illustrate the working of Markovian EM agents. The evaluation of MR brain scan segmentation was performed using both phantoms and real $3 \mathrm{~T}$ brain scans. It showed a robustness to intensity non-uniformity and noise, together with a low computational time.

Conclusion: Based on these experiments MRF agent-based approach appears to be a very promising new tool for complex image segmentation.

(C) 2008 Published by Elsevier B.V.

\section{Introduction}

The Markov random field (MRF) probabilistic framework is a powerful tool for image segmentation. It introduces spatial dependencies between labels, providing a labeling regularization and a robust-tonoise segmentation. Spatial dependencies however lead Markov model-based segmentation to an extremely large amount of computation for the estimation of the model parameters with a standard expectation-maximization (EM) algorithm. Variants of $E M$ have been proposed to make the MRF case tractable. Classically, model parameter estimation is performed over the whole image, and does not reflect local image properties. We propose to agentify the global MRF segmentation model by distributing a set of local MRF models within a multiagent framework. The image is partitioned in territories where situated agents estimate the parameters of a local MRF model. The global consistency of local models is ensured by modifying the EM scheme to introduce cooperation between neighboring agents. Local models estimated with our distributed local EM (DILEM) then accurately fit local image features. A priori knowledge can also be integrated into this model via the external field of MRF models, and can be used to introduce other levels of agent cooperation.

We illustrate the agentified Markovian EM procedure on magnetic resonance (MR) brain scan segmentation. These images are difficult to segment due to a number of artifacts, leading to low contrast between tissues, high level of noise with strong field images (3 $\mathrm{T}$ or higher) or intensity non-uniformity. We distribute local non-overlapping agents to perform tissue segmentation. They estimate, via our DILEM algorithm, the MRF parameters for white matter (WM), grey matter (GM) and cephalo-spinal fluid (CSF) classes. In addition, we perform subcortical structure segmentation by distributing local agents that focus on some interesting brain regions. Structure segmentation is not reduced to a postprocessing step but cooperates with tissue segmentation so that they mutually improve. With our approach, a multilevel regularization is thus introduced: regularization at the voxel level via MRF modelization, at a territory level via the DILEM algorithm, and at a regional level via the local tissue-structure cooperations.

We detail how to design such a MRF agent-based segmentation approach and report several experiments that illustrate the working of our multiagent framework. The evaluation of MR brain scan segmentation was performed using both phantoms and real $3 \mathrm{~T}$ brain scans. We show that the agentified MRF segmentation framework provides interesting properties for such complex image segmentation: (1) the use of MRF makes the segmentation robust to noise, (2) since intensity models are estimated locally it does not require the explicit modelization of nonuniformity as required by classical approaches $[1,2]$ and (3) the approach is time efficient compared to classical MR brain scan segmentation tools. This MRF agent-based approach extends our previous work $[3,4]$ and appears as a very promising new tool for complex image segmentation.

This paper is organized as follows. In Section 2 we present the MRF segmentation framework and the classical EM scheme for parameter estimation. We then show in Section 3 how MRF processes are turned into cooperating agent entities and how parameters are estimated with our DILEM algorithm. In Section 4 we apply our agentified MRF segmentation to tissue and structure segmentation on MR brain scan. Section 5 reports evaluation results and presents several experiments to exhibit some interesting properties of such an agentified approach. Section 6 is devoted to discussion and conclusion.

\section{MRF agent-based segmentation}

\subsection{MRF framework}

The Markovian segmentation framework [5] is commonly used in the field of segmentation. It introduces spatial dependencies between voxels, providing a robust-to-noise segmentation. We consider a finite set of $N$ sites $S=\{1, \ldots, N\}$. A typical example in image analysis is the regular two-dimen- 
sional grid of pixels (picture elements) for twodimensional image segmentation or the regular three-dimensional grid of voxels (volume elements) for volume segmentation. $S$ is related to a neighborhood system where $\mathcal{N}(i)$ denotes the set of sites neighboring $i$. Our aim is to assign each site $i$ to one of $K$ classes considering the observed features data $y_{i}$ at site $i$. In the case of greylevel image segmentation $y_{i} \in\{R\}$ is the observed greylevel intensity at site $i$. Both observed intensities and hidden labels are considered to be a random field (a collection of random variables) denoted, respectively by $Y=\left\{Y_{1}, \ldots, Y_{N}\right\}$ and $Z=\left\{Z_{1}, \ldots\right.$, $\left.Z_{N}\right\}$; the bold notation being used to indicate a collection of variables. Each random variable $Z_{i}$ takes its value in $\left\{e_{1}, \ldots, e_{k}\right\}$ where $e_{k}$ is a $K$ dimensional binary vector corresponding to class $k$. Only the $k^{\text {th }}$ component of this vector is nonzero and is set to $1 . z=\left\{z_{1}, \ldots, z_{N}\right\}$ is a configuration of $Z$ corresponding to a realization of the random field. Hidden data $\mathbf{Z}$ are described by a discrete Markov random field whose joint distribution probability satisfies by definition of the following properties:

$\forall \mathbf{z}, \quad P_{G}\left(z_{i} \mid \mathbf{z}_{S \backslash\{i\}},\right)=p\left(z_{i} \mid z_{j}, \quad j \in \mathcal{N}(i)\right)$

$\forall \mathbf{z}, \quad P_{G}(\mathbf{z})>0$,

where $\mathbf{z}_{\mathrm{S} \backslash\{i\}}$ denotes a realization of the field restricted to $S \backslash\{i\}=\{j \in V, j \neq i\}$. Property (1) means that the interactions between site $i$ and the other sites are reduced to interactions with its neighbors, introducing spatial dependencies between voxels. Property (2) is important for the Hammersley-Clifford theorem to hold. This theorem states that the joint probability distribution of a Markov field, which depends on some parameters $\Phi_{z}$, is a Gibbs distribution given by

$P_{G}\left(\mathbf{z} \mid \Phi_{z}\right)=W_{\Phi_{z}}^{-1} \exp \left(-H\left(\mathbf{z} \mid \Phi_{z}\right)\right)$,

where $W_{\Phi_{z}}=\sum_{\mathbf{z}^{\prime}} \exp \left(-H\left(\mathbf{z}^{\prime} \mid \Phi_{z}\right)\right)$ is a normalization constant. When assuming in addition that the observations $Y$ are conditionally independent given $Z$ and depend on some parameters $\Phi_{y}$ we show using the Bayes rule that the conditional field $Z$ given $Y=y$ is also a Markov field with energy function given by

$H(\mathbf{z} \mid \mathbf{y}, \Phi)=H\left(\mathbf{z} \mid \Phi_{z}\right)-\sum_{i \in S} \log p\left(y_{i} \mid z_{i}, \Phi_{y}\right)$,

with $\Phi=\left(\Phi_{y}, \Phi_{z}\right)$. This central energy in Markov random field segmentation models is composed of two terms:

- The first term of (3) is a regularization term that accounts for spatial dependencies between voxels. They are several definition of $H\left(\mathbf{z} \mid \Phi_{z}\right)$ in the literature of image segmentation. In this work we will consider a Potts model with external field. In the following definition $t$ denotes the transpose operator and for two vectors $z_{i}$ and $v_{i}, z_{i}^{t} \cdot v_{i}$ denotes the scalar product:

$H\left(\mathbf{z} \mid \Phi_{z}\right)=\sum_{i \in S}\left[z_{i}^{t} \cdot v_{i}-\frac{\beta}{2} \sum_{j \in \mathcal{N}(i)} v_{i j}\left(z_{i}, z_{j}\right)\right]$.

$V_{i j}\left(z_{i}, z_{j}\right)$ introduces spatial interactions between voxels $i$ and $j$. It tends to favor neighbors that are in the same class when $\beta$ is positive. It is classically modeled by $V_{i j}\left(z_{i}, z_{j}\right)=z_{i}^{t} \cdot z_{j}$ so as $V_{i j}\left(z_{i}, z_{j}\right)=1$ when $z_{i}$ and $z_{j}$ are in the same class. Other approaches introduce voxel interactions weighted by their distance. $\beta$ is a parameter that accounts for the strength of spatial interaction. The parameters $v_{i}{ }^{\prime} \mathrm{s}$ are $K$-dimensional vectors defining the so-called external field. In this case $\Phi_{\mathrm{z}}=\left\{v_{1}, \ldots, v_{\mathrm{N}}, \beta\right\}$.

- The second term of (3) is the data-driven term based on intensities. We generally consider a Gaussian probability density function of observed intensity $y_{i}$ for class $z_{i}$. It follows that $p\left(y_{i} \mid z_{i}=\right.$ $\left.e_{k}, \Phi_{y}\right)=g_{\mu_{k}, \sigma_{k}}\left(y_{i}\right)$ with $\Phi_{y}=\left\{\mu_{k}, \sigma_{k}, k=1, \ldots, K\right\}$.

\subsection{Global EM procedure for the estimation of MRF parameters}

Segmentation is performed according to the maximum a posteriori principle (MAP) by maximizing over $\mathbf{z}$ the probability:

$P_{\mathrm{G}}(\mathbf{z} \mid \mathbf{y}, \Phi)=W_{\mathbf{y}, \Phi}^{-1} \exp (-H(\mathbf{z} \mid \mathbf{y}, \Phi))$.

This requires the evaluation of an intractable normalizing constant $W_{y, \Phi}$ and the estimation of the unknown parameters $\Phi$. A standard approach is to use the iterative conditional modes (ICM) algorithm [6] that alternates between parameter estimation and segmentation but results in biased estimates. We rather consider EM-based algorithms and use variants proposed by [7].

Assuming $\Phi$ unknown, the aim is to get the maximum likelihood estimate of this parameter knowing the observations y. The log-likelihood of the model is $L(\Phi)=\log P_{G}(\mathbf{y} \mid \Phi)=\log \sum_{\mathbf{z}} P_{G}(\mathbf{y}, \mathbf{z} \mid \Phi)$.

The EM algorithm [8] is an iterative algorithm aiming at maximizing this log-likelihood by maximizing at iteration $q$,

$Q\left(\Phi \mid \Phi^{(q)}\right)=I E_{\Phi^{(q)}}\left[\log P_{\mathrm{G}}(\mathbf{y}, \mathbf{Z} \mid \Phi) \mid \mathbf{Y}=\mathbf{y}\right]$,

the expectation of the complete log-likelihood knowing the observation $\mathbf{y}$ and current estimate $\Phi^{(q)}$. The EM algorithm can therefore be described as follows: 

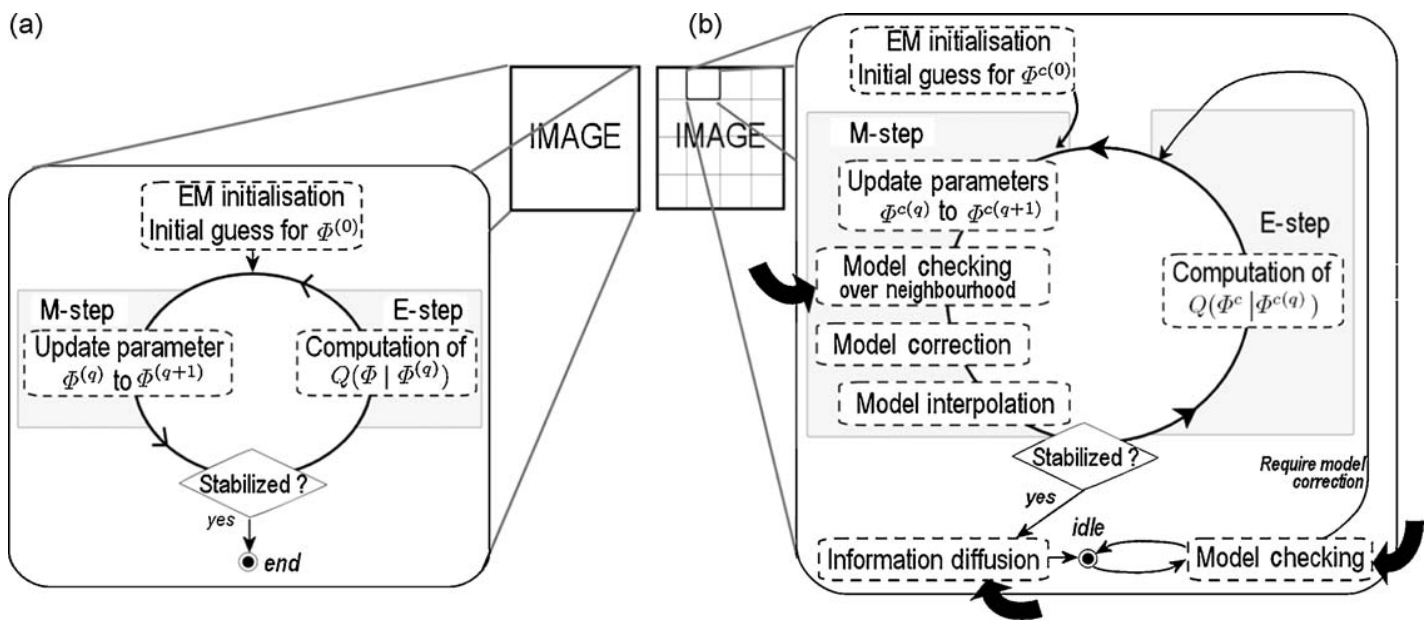

Figure 1 (a) Shows a synthetic view of a classical global EM estimation scheme. (b) Shows the agentified distributed local EM (DILEM) scheme with cooperations and coordinations (bold arrows) to ensure a global consistency of local models.

(1) start from an initial guess $\Phi^{(0)}$ for $\Phi$,

(2) update the current estimate $\Phi^{(q)}$ to $\Phi^{(q+1)}$ $=\arg \max _{\Phi} Q\left(\Phi \mid \Phi^{(q)}\right)$.

The updating part (2) can be divided in two steps. The computation of $Q\left(\Phi \mid \Phi^{(q)}\right)$ corresponds to the $\mathrm{E}$ (expectation) step and the maximization with respect to $\Phi$ to the $M$ (maximization) step. However, the spatial dependencies of MRF models lead to an extremely high combinatory complexity with a standard EM procedure. Celeux et al. [7] proposed mean-field approximations to make the EM algorithm tractable for the MRF case. The principle is to approximate at each iteration the MRF model with complex dependencies by a system of independent variables. It is obtained by fixing the neighborhood of each pixel to arbitrary constants. Celeux et al. [7] proposed three algorithms referred to as mean-field, modal-field and simulated-field algorithms based on different mean-field approximations via the use of different constants.

With these approaches one unique and global EM procedure estimates the MRF parameters describing the whole image (see Fig. 1a), which does not reflect local properties of the image. In the next section we propose to agentify the EM procedure in order to estimate local parameters through the volume.

\section{Agentification of EM procedures}

We propose to agentify the global MRF segmentation model by distributing in the volume several local MRF models in a multiagent (MA) paradigm. This agentification modifies the standard EM procedure (see Fig. 1a) and leads to the introduction of additional steps and agent interactions (see Fig. 1b).
We consider a decentralized and memory shared MA framework based on the classical Agent/Group/Behavior conceptual model inspired from MadKit. ${ }^{1}$ In this model an agent is an autonomous and communicating entity which runs behaviors within groups. Each agent can handle multiple behaviors but only one is active at a time. Groups are defined as atomic sets of agent aggregation and thus gather cooperating or communicating agents in the system. An agent can be a member of $n$ groups at the same time and groups can freely overlap.

\subsection{Hierarchical setup of the agents}

We consider two kinds of agents: one global agent which initializes the MRF agentified segmentation and a set of local cooperating MRF segmentation agents.

\subsubsection{Global agent}

At the system launch time, the global agent $\mathcal{A}_{G}$ is created and activated. This agent belongs to only one group $\mathcal{G}_{\text {ALL_AGENTS }}$ that gathers all agents of the system and runs two consecutive behaviors:

- $\mathcal{B}_{\text {GlbAgent_Partitionning: This behavior is activated }}$ first. It is responsible for partitioning the image into $C$ non-overlapping territories $\left\{\mathcal{T}_{c}, C=\right.$ $1, \ldots, C\}$ and instantiating one local segmentation agent $\mathcal{A}_{c}$ per territory. The global agent then enters in idle mode and waits for the local agents to finish their initialization before running the $\mathcal{B}_{\text {GlbAgent_WakeUpAgents }}$ behavior.

\footnotetext{
${ }^{1}$ See the MadKit homepage: http://www.madkit.org (accessed: 6 August 2008).
} 
- $\mathcal{B}_{\text {GlbAgent_WakeUpAgents: This behavior is activated to }}$ initiate the agentified segmentation when each local segmentation agent $\mathcal{A}_{c}$ has finished its initialization (see Section 3.2).

\subsubsection{Local EM agents}

Each agent $\mathcal{A}_{c}$ is a situated agent attached to its territory $\mathcal{T}_{c}$. Its role is to estimate over $\mathcal{T}_{c}$ the parameters of a local MRF model via a local EM procedure.

For each $\mathcal{A}_{c}$ the MRF model $\mathcal{M R \mathcal { F }}{ }_{c}$ is defined by the energy (see Eqs. (3) and (4)):

$$
\begin{aligned}
& H^{c}\left(\mathbf{z} \mid \mathbf{y}, \Phi^{c}\right)= \\
& \sum_{i \in \mathcal{T}_{c}^{T}}\left[z_{i}^{t} \cdot \lambda_{i}^{c}-\frac{\beta^{c}}{2} \sum_{j \in \mathcal{N}(i)} V_{i j}\left(z_{i}, z_{j}\right)-\log p\left(y_{i} \mid z_{i}, \Phi_{y}^{c}\right)\right],
\end{aligned}
$$

where the parameters $\Phi^{c}=\left\{\Phi_{t}^{c}, \Phi_{y}^{c}\right\}$ are local to the territory $\mathcal{T}_{c}$. Each agent $\mathcal{A}_{c}$ owns three behaviors:

- $\mathcal{B}_{\text {LocalAgent_Init }}$ which initializes the EM algorithm by computing initial intensity models via a fuzzy cmean (FCM) algorithm,

- $\mathcal{B}_{\text {LocalAgent_DILEMLoop }}$ which estimates the local MRF model parameters in cooperation with neighboring agents and

- $\mathcal{B}_{\text {LocalAgent_Stabilized }}$ which activates the neighboring agents (knowledge diffusion) and then waits to be activated by its neighbors.

\subsection{Interaction between agents}

Several coordination and cooperation mechanisms are proposed to realize interaction between agents.

\subsubsection{Coordination between the global and the local agents}

When all local agents have finished their initialization the global agent runs its $\mathcal{B}_{\text {GlbAgent_WakeUpAgents }}$ behavior (see Fig. 2). First it computes a simple global intensity model without any Markov regularization using the FCM algorithm. Then, it initiates the agentified segmentation procedure by activating a first subset of local agents. We use the strategy of activating only agents with initial local models closest to the global model because they are likely to start with a more reliable knowledge than others. Other agents will be activated by coordination mechanisms between local neighboring agents.

\subsubsection{Cooperation between neighboring agents}

Because the estimation is local, some classes are likely to be under-represented in some territories, leading to poor model estimations with a classical EM scheme. The agent $\mathcal{A}_{c}$ thus cooperates with its neighbors to ensure a global consistency of the local estimated model. We denote by $\mathcal{G}_{\mathcal{N}\left(\mathcal{A}_{c}\right)}$ the group of agents neighboring $\mathcal{A}_{c}$ and provide details about the $\mathcal{B}_{\text {LocalAgent_DILEMLoop }}$ and $\mathcal{B}_{\text {LocalAgent_Stabilized }}$ behaviors (see Fig. 1b): we modify the classical EM scheme by introducing model checking, model correction and model interpolation as described below:

- Model checking: For each class $k$ the agent $\mathcal{A}_{c}$ computes an intensity model averaging the intensity models of its neighbors $\mathcal{G}_{\mathcal{N}}\left(\mathcal{A}_{c}\right)$. This average model is denoted by $\Phi_{y, k}^{\tilde{c}}=\left\{\tilde{\mu}_{k}^{c}, \sigma_{k}^{c}\right\}$.

Then $\mathcal{A}_{c}$ compares its local intensity model $\left\{\mu_{k}^{c}, \sigma_{k}^{c}\right\}$ to $\left\{\tilde{\mu}_{k}^{c}, \tilde{\sigma}_{k}^{c}\right\}$ using the Kullback-Leibler

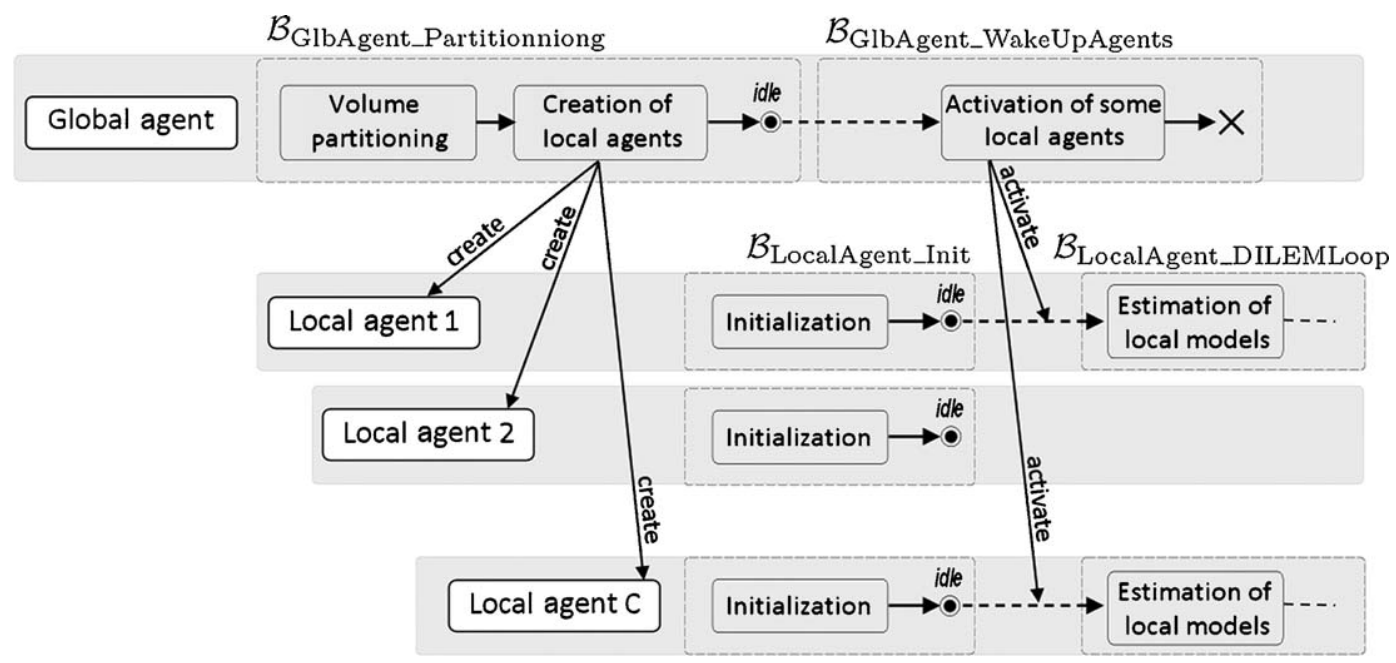

Figure 2 Interactions between the global and local agents at the initialization of the agentified MRF segmentation. 


$$
\begin{aligned}
& \text { distance given by } \\
& K L\left(\boldsymbol{g}_{\mu_{1}, \sigma_{1}}, \boldsymbol{g}_{\mu_{2}, \sigma_{2}}\right)= \\
& \qquad \frac{\left(\sigma_{1}^{2}-\sigma_{2}^{2}\right)^{2}+\left(\mu_{1}-\mu_{2}\right)^{2}\left(\sigma_{1}^{2}+\sigma_{2}^{2}\right)}{4 \sigma_{1}^{2} \sigma_{2}^{2}}
\end{aligned}
$$

Thus, $\mathcal{D}_{k}^{c}=K L\left(g_{\mu_{k}^{c}, \sigma_{k}^{c}}, \boldsymbol{g}_{\mu_{k}^{c}} \tilde{\sigma_{c}^{c}}\right)$ provides a measure of dissimilarity between the two intensity models of class $k$.

- Model correction: We assume that for each class $k$ the local model of $\mathcal{A}_{c}$ is likely to be close to the local mean model. We compute the corrected mean $\widehat{\mu_{k}^{c}}$ and variance $\widehat{\sigma_{k}^{c}}$ of class $k$ from a linear combination of intensity models $\left\{\mu_{k}^{c}, \sigma_{k}^{c}\right\}$ and $\left\{\tilde{\mu}_{k}^{c}, \tilde{\sigma}_{k}^{c}\right\}$ :

$\widehat{\mu_{k}^{c}}=(1-\kappa) \mu_{k}^{c}+\kappa \tilde{\mu_{k}^{c}}$

$\widehat{\sigma_{k}^{c}}=(1-\kappa) \sigma_{k}^{c}+\kappa \tilde{\sigma_{k}^{c}}$,

with $\kappa \in[0,1]$. We define two thresholds $\delta_{\text {keep }}$ and $\delta_{\text {replace }}$ with $\delta_{\text {keep }} \leq \delta_{\text {replace }}$ and propose the following strategy of model correction:

o If $\mathcal{D}_{k}^{c} \leq \delta_{\text {keep }}$, the estimated local model of class $k$ is considered correct and we keep it: $\kappa=0$.

o If $\mathcal{D}_{k}^{c}>\delta_{\text {replace }}$ we replace the estimated model by the local mean model of class $k: \kappa=1$.

o Else, if $\delta_{\text {keep }}<\mathcal{D}_{k}^{c} \leq \delta_{\text {replace }}$, we partially correct the estimated model with a linear interpolation:

$$
\kappa=\frac{\mathcal{D}_{k}^{c}-\delta_{\text {keep }}}{\delta_{\text {replace }}-\delta_{\text {keep }}}
$$

- Model interpolation: Model correction provides a corrected intensity model, namely $K$ Gaussians, to describe the local intensity distribution in $\mathcal{T}_{c}$. Then we compute one intensity model per voxel by using cubic splines interpolation between corrected models of $\mathcal{A}_{c}$ and of $\mathcal{G}_{\mathcal{N}\left(\mathcal{A}_{c}\right)}$. It follows that $\Phi_{y}^{c}=\left\{\mu_{k, i}, \sigma_{k, i}, \quad k=1, \ldots, K, \quad i \in V_{c}\right\}$. This results in a non-stationary field-like approach. There is not a unique mixture of Gaussians describing the intensity distribution over the territory $\mathcal{T}_{c}$ but one mixture of Gaussians per voxel. It has the advantage to ensure smooth model variation between neighboring territories. In the following, since territories are not overlapping, we will denote by $\Phi_{i y}=\left\{\mu_{k, i}, \sigma_{k, i}, k=1, \ldots, K\right\}$ the parameters of the mixture of Gaussians for site $i \in S$.

\subsubsection{Coordination between neighboring agents}

When the agent $\mathcal{A}_{c}$ has finished its local model estimation it enters in $\mathcal{B}_{\text {LocalAgent_Stabilized }}$ behavior (see Fig. 3). This behavior first activates neighboring agents $\mathcal{G}_{\mathcal{N}\left(\mathcal{A}_{c}\right)}$ so that they perform estimation in turn and then put $\mathcal{A}_{c}$ in idle mode. Two cases are possible after the activation of $\mathcal{A}_{c^{\prime}} \in \mathcal{G}_{\mathcal{N}\left(\mathcal{A}_{c}\right)}$ by $\mathcal{A}_{c}$ :

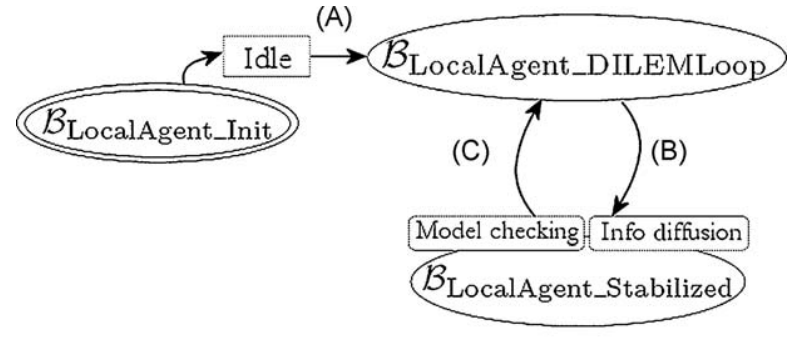

(A) : Activation by the global agent or by a neighbouring agent

(B) : After convergence of the local EM algorithm

(C) : Activation by a neighbouring agent

Figure 3 State diagram for the behaviors execution of a local agent.

- $\mathcal{A}_{c^{\prime}}$ had already completed its estimation before and is in $\mathcal{B}_{\text {LocalAgent_Stabilized behavior. In this case }}$ $\mathcal{A}_{c^{\prime}}$ performs model checking. When model correction is required, the corresponding

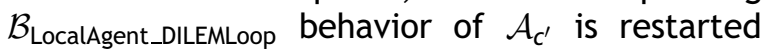
to take into account the model modifications (see Fig. 3). We define a maximum number $\mathrm{MAX}_{\text {AgentRestart }}$ to ensure the convergence of the algorithm.

- $\mathcal{A}_{c^{\prime}}$ has only performed its initialization. In this case it enters in $\mathcal{B}_{\text {LocalAgent_DILEMLoop }}$ to compute a first estimate of the local MRF model.

\subsubsection{Extended cooperation for overlapping agents}

Agents can also cooperate via the external field of the MRF model. By considering the Potts model (see Eq. (4)) we notice that when $\beta=0$ (no spatial interaction between sites) it comes:

$P_{G}\left(z_{i}=e_{k} \mid \Phi_{z}\right)=\frac{\exp \left(-v_{i}(k)\right)}{\sum_{l=1}^{K} \exp \left(-v_{i}(l)\right)}$,

so that $v_{i}$ can be related to a priori weights accounting for the relative importance of the $K$ classes at site $i$. The introduction of these extra parameters in the standard Potts model is seldom considered in MRF approaches whereas it makes possible the integration of a priori knowledge for each site in the segmentation procedure. Two overlapping agents $\mathcal{A}_{c}$ and $\mathcal{A}_{c^{\prime}}$ can therefore cooperate by sharing data via the external field: if $\mathcal{A}_{c}$ has a reliable knowledge about the shared sites with $\mathcal{A}_{c^{\prime}}$, it can introduce it as a priori weights in the model of $\mathcal{A}_{c^{\prime}}$. We describe in Section 4.2 an example of such a cooperation.

\section{Markovian EM procedure applied to MR brain scans}

Our agent-based approach provides a two-level regularization during the segmentation: a regularization 
at the label level via MRF models and a regularization at the territory level via cooperations and coordinations between agents. These mechanisms provide robust local models that better fit to local image features compared to a global model. Such an approach is then particularly interesting in presence of spatial intensity non-uniformity. It intrinsically handles the variations of intensity inside classes without any specific modelization. The only assumption is that intensity non-uniformity varies smoothly over the image. In addition local agentified segmentation takes advantage of local complexity in the image: easy to segment territories lead to a fast EM stabilization, allowing the system to focus on more difficult territories. It is then especially adapted to MRF segmentation known to be time consuming.

We assume that such properties are well suited to the segmentation of specific images such as MR brain scans. Indeed, MR brain scans are corrupted by a number of artifacts such as noise and intensity nonuniformity that make the segmentation a challenging task. The intensity non-uniformity results in spatial intensity variations within each tissue class. It results mainly from MR imaging hardware imperfections, such as magnetic field non-uniformity or receiver coil imperfections, and from biological tissue properties. Even if it does not really affect visual perception, the non-uniformity of image intensity is a major obstacle to an accurate automatic segmentation. In the MR brain scan segmentation field, the data are three-dimensional data referred as volumes composed of voxels (volume element), and we generally consider:

- The tissue segmentation, that aims at classifying voxels in $K=3$ tissue classes: CSF, GM, and WM, and

- The subcortical structure segmentation, subcortical structures being regions of tissues (see Fig. 5a) known to be involved in various brain functions. Their segmentation is very interesting for various neuroanatomical researches such as brain development or disease progression studies.

We propose to use our local MRF agent-based approach to build the local cooperative unified segmentation (LOCUS) tool $[9,10]$. Global Markovian modelization have been widely used for a robust-tonoise segmentation of MR brain scans. Earlier approaches used ICM to estimate MRF parameters [11] that is known to result in biased estimates. More recent approaches introduced variants of the EM algorithms [12,13] for a more reliable estimation. They estimate parameters through the entire volume and then require the estimation of an explicit "bias field" model [1] to account for the inten- sity non-uniformity. These models are based on underlying assumptions that are not always valid and requires additional computational burden for their estimation. For all segmentation techniques tissue and structure segmentations are considered as two successive tasks whereas they are clearly linked: the knowledge of structure segmentation provides valuable information about local intensity distribution for a given tissue, and should be introduced in the tissue segmentation model. In the following sections we apply our agentified MRF segmentation framework to propose a cooperative tissue and structure segmentation approach.

\subsection{Tissue segmentation agents}

The agentified Markovian EM approach estimates local parameters through the volume. This results in a good model adaptation to local image properties and therefore does not require non-uniformity correction as seen on Fig. 4. We then apply the agentified DILEM scheme exactly as shown in Section 3. We denote by $\mathcal{A}_{G}^{T}$ the global tissue segmentation agent and by $\mathcal{A}_{c}^{\top}$ the local tissue segmentation agents. The agent territory $\mathcal{T}_{c}^{T}$ is a three-dimensional subvolume of voxels. We denote by $t=$ $\left\{t_{1}, \ldots, t_{N_{c}}\right\}$ the hidden tissue classes at each voxel. ti's take their values in $\left\{e_{1}, e_{2}, e_{3}\right\}$, respectively for classes $\left\{e_{\mathrm{CSF}}, e_{\mathrm{GM}}, e_{\mathrm{WM}}\right\}$.

\subsection{Structure segmentation agents}

We propose to couple tissue and structure segmentation so as they mutually improve.

\subsubsection{Agent modelization}

We define one structure agent $\mathcal{A}_{l}^{S}$ per structure and currently consider $L=9$ subcortical structures: the ventricular system, the two frontal horns, the two

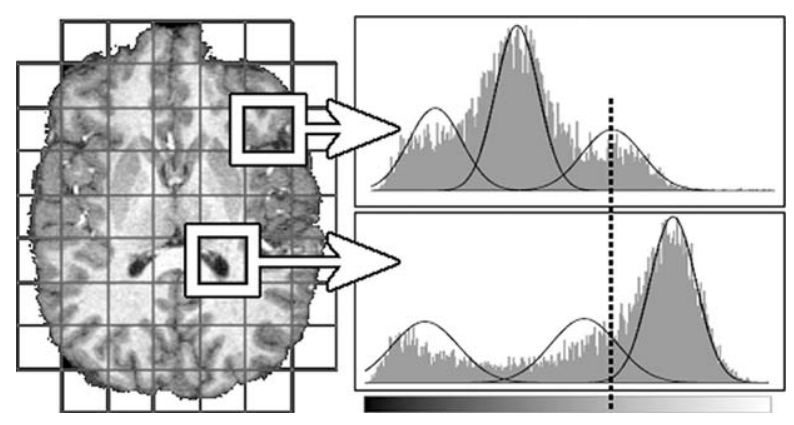

Figure 4 The two histograms corresponding to two different three-dimensional territories illustrate the intensity variation for each tissue class due to intensity non-uniformity. Local estimated models fit to local image features: the vertical bar shows a greylevel intensity labeled either as WM or GM according to the subvolume. 

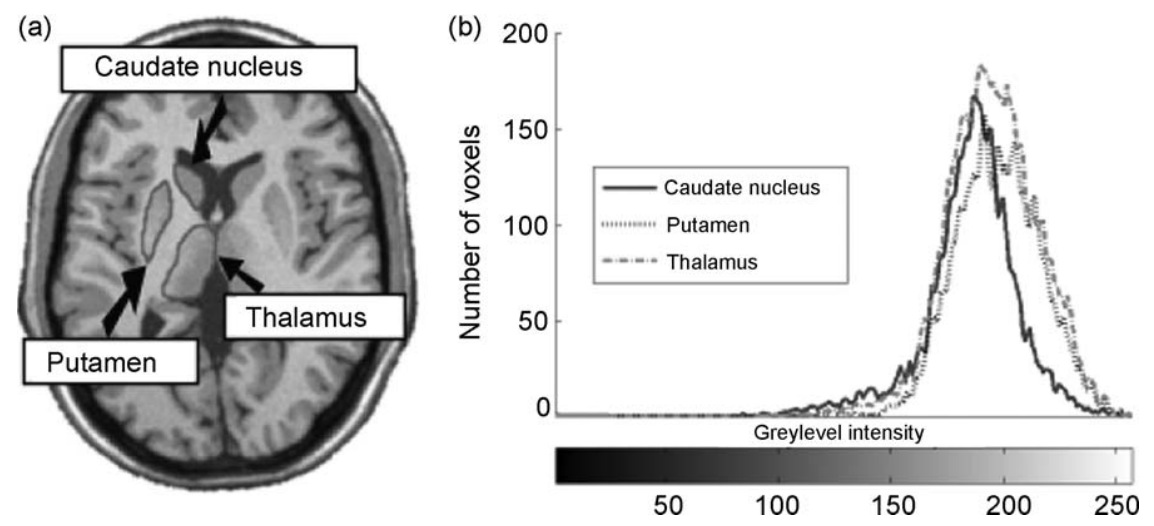

Figure 5 Manual delineation of some subcortical structures (a) and corresponding intensity histograms (b). It illustrates the inherent difficulty to automatically label these structures based only on greylevel radiometry.

caudate nucleus, the two thalamus, and the two putamens. Each structure agent segments its territory $\mathcal{T}_{l}^{S}$ with a MRF model of $K=2$ classes referred as structure and background. Denoting by $\mathbf{s}=$ $\left\{s_{i}, \quad i \in \mathcal{T}_{l}^{S}\right\}$ the hidden classes, the energy function of the MRF model of $\mathcal{A}_{l}^{S}$ is given by

$H^{l}\left(\mathbf{s} \mid \mathbf{y}, \Psi^{l}\right)=$

$$
\sum_{i \in \mathcal{T}_{l}^{S}}\left[s_{i}^{t} \cdot \alpha_{i}^{l}-\frac{\beta^{l}}{2} \sum_{j \in \mathcal{N}(i)} V_{i j}\left(s_{i}, s_{j}\right)-\log p\left(y_{i} \mid s_{i}, \Psi_{y}^{l}\right)\right],
$$

with $\Psi^{l}=\left\{\Psi_{s}^{l}, \Psi_{y}^{l}\right\}$ and $s_{i} \in\left\{e_{1}, e_{2}\right\}=\left\{e_{\mathrm{B}}, e_{\mathrm{s}}\right\}$ for a voxel of the background or a voxel belonging to structure $l$.

\subsubsection{Structure segmentation requires introduction of a priori knowledge}

Automatic structures segmentation cannot rely only on radiometry information because intensity distributions of grey nucleus are largely overlapping (see Fig. 5b). A priori knowledge should be introduced. Classical approaches rely on an a priori known atlas describing anatomical structures. Atlas warping methods are however time consuming and limited due to inter-subject variability. A recent different way to introduce a priori anatomical knowledge is to describe brain anatomy with generic fuzzy spatial relations $[14,15]$. We generally consider three kind of spatial relations: distance, symmetry and orientation relations. They are expressed as 3D fuzzy maps to take into account the general nature of the provided knowledge (see Fig. 6). We describe each subcortical structure by a set of generic fuzzy spatial relations provided by a brain anatomist. Fusion operators between fuzzy sets then permits to combine the knowledge provided by each spatial relation and provides a fuzzy localization map (FLM) of the structure in the volume.

The FLM $f^{l}$ of structure $l$ is used in two ways: first it dynamically provides the structure agent territory

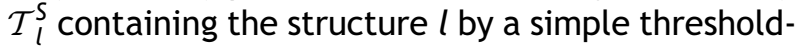
ing. Second we propose to integrate it as an a priori anatomical knowledge in the MRF framework via the external field denoted by $\left\{\alpha_{i}, \quad i \in \mathcal{T}_{l}^{S}\right\}$ (see Eq. (7)), where $\alpha_{i}^{l}$ is a two-dimensional vector. We denote by $f_{i}^{l}$ the value of $f^{l}$ at voxel $i$ and propose to introduce the prior fuzzy knowledge of the FLM as relative prior weights for each voxel $i$, by setting:

$\alpha_{i}^{l}=\gamma\left[\begin{array}{l}-\log \left(1-f_{i}^{l}\right) \\ -\log f_{i}^{l}\end{array}\right]$,

where $\gamma$ adjusts the influence of the external field. When $f_{i}^{l} \approx 0$, the voxel $i$ is unlikely to belong to the structure. If $\gamma$ was null, the segmentation would be performed only from the intensity models. Else, according to (8), $\alpha_{i}^{l}(1)<\alpha_{i}^{l}(2)$ which favours in (7) the class background. When $f_{i}^{l} \approx 1$, the voxel $i$ is (a)

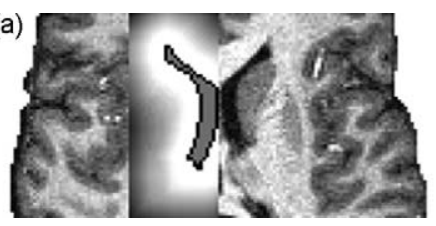

(b)

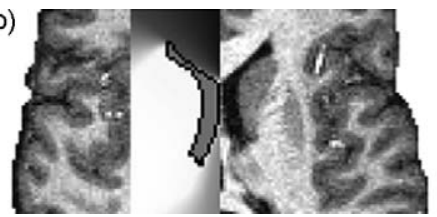

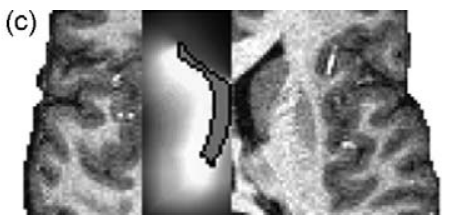

Figure 6 Example of fuzzy maps of spatial relations for the left caudate nucleus: (a) express "approximatively at $5 \mathrm{~mm}$ from the left frontal horn", (b) express "in a direction $(-3 \pi / 4, \pi / 2)$ in the Talairach system with respect to the left frontal horn", and (c) is the fusion of the two maps. 
likely to belong to the structure. In that case $\alpha_{i}^{l}(1)>\alpha_{i}^{l}(2)$ and the class structure is favored.

\subsubsection{Structure agent behaviors}

Structure agents owns four behaviors:

- $\mathcal{B}_{\text {Struct_nitialization that initializes the structure }}$ agent,

- $\mathcal{B}_{\text {Struct_Computefuzzymap }}$ that computes or updates the FLM from fuzzy spatial relations,

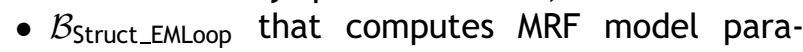
meters via an EM loop and

- $\mathcal{B}_{\text {Struct_Stabilized }}$ when the agent is in the idle state.

\subsection{Cooperation and coordination between agents}

As we defined a neighborhood group $\mathcal{G}_{\mathcal{N}\left(\mathcal{A}_{\mathcal{C}}\right)}$ for each tissue agent we introduce the following groups:

- $\mathcal{G}_{T \rightarrow S\left(\mathcal{A}_{1}^{S}\right)}$ of tissue agents cooperating with a structure agent $\mathcal{A}_{l}^{S}$, i.e. tissue agents overlapping $\mathcal{A}_{l}^{S}$,

- $\mathcal{G}_{S \rightarrow T\left(\mathcal{A}_{C}^{T}\right)}$ of structure agents cooperating with a tissue agent $\mathcal{A}_{c}^{T}$, i.e. structure agents overlapping $\mathcal{A}_{c}^{T}$ and

- $\mathcal{G}_{S \rightarrow S\left(\mathcal{A}_{l}^{S}\right)}$ of structure agents using $\mathcal{A}_{l}^{S}$ as a reference in a spatial relation.

These groups allow to detail cooperation and coordination mechanisms between agents.

\subsubsection{Updating structure models via tissue models}

Each structure being composed of a single tissue $T^{l} \in\left\{e_{\mathrm{CSF}}, e_{\mathrm{GM}}, e_{\mathrm{WM}}\right\}$, we do not estimate intensity model $\Psi_{y}^{l}$ of class structure and class background. We rather compute them from tissue intensity models of $\mathcal{G}_{T \rightarrow S\left(\mathcal{A}_{l}^{S}\right)}$ estimated by tissue agents, by setting:

$$
\left\{\begin{array}{l}
p\left(y_{i} \mid s_{i}=e_{S}, \Psi_{y}^{l}\right)=p\left(y_{i} \mid t_{i}=T^{l}, \Phi_{i y}\right) \\
p\left(y_{i} \mid s_{i}=e_{B}, \Psi_{y}^{l}\right)=\max _{l \in \mathcal{G}_{S \rightarrow T\left(\mathcal{A}_{c}^{T}\right)}, T^{l}=e_{k}} p\left(y_{i} \mid t_{i}=t, \Phi_{i y}\right),
\end{array}\right.
$$

so that improvements in tissue intensity models estimation will be dynamically taken into account by structure agents.

\subsubsection{Feedback of structure segmentation on tissue segmentation}

Conversely, results from structure agents are integrated in the tissue segmentation model via the external field $\lambda_{i}^{c}=\left[\lambda_{i}^{c}\left(e_{C S F}\right), \lambda_{i}^{c}\left(e_{G M}\right), \lambda_{i}^{c}\left(e_{W M}\right)\right]$, of tissue agents. The tissue agent $\mathcal{A}_{c}^{T}$ introduces in its model the posteriori probabilities of overlapping structure agents $\mathcal{G}_{S \rightarrow T\left(\mathcal{A}_{c}^{T}\right)}$ as a priori weights for each tissue class. More specifically we use for tissue class $e_{k}$ the weight:

$\lambda_{i}^{c}\left(e_{k}\right)=-\log \left(\max _{l \in \mathcal{G}_{s \rightarrow T\left(\mathcal{A}_{c}^{T},\right.}, T^{l}=e_{k}} p\left(s_{i}^{l}=e_{s} \mid \mathbf{y}, \Psi^{l}\right)\right)$,

so that the tissue class $e_{k}$ will be favored when voxel $i$ belong to a structure composed of tissue $e_{k}$. It follows that structure segmentation is not reduced to a second step but is combined with tissue segmentation to improve its performance. It thus introduces a regularization mechanism between the two different knowledge.

\subsubsection{Update fuzzy maps}

When the segmentation of structure $l$ is updated the structure models of $\mathcal{G}_{S \rightarrow s}\left(\mathcal{A}_{l}^{S}\right)$ take it into account by re-computing their spatial relations with respect to l, making the knowledge gradually more accurate.

\subsubsection{Activating tissue agents}

Each time the convergence of a structure agent is reached, it activates corresponding cooperative tissue agents of $\mathcal{G}_{T \rightarrow S\left(\mathcal{A}^{S}\right)}$ so that they take into account the improved knowledge.

\subsubsection{Activating structure agents}

When the convergence of a tissue agent is reached, it activates corresponding structure agents of $\mathcal{G}_{S \rightarrow T\left(\mathcal{A}_{c}^{T}\right)}$ only if tissue intensity models have sufficiently changed. This threshold permits the algorithm to converge.

Fig. 7 shows a global view of LOCUS approach for cooperative tissue and structure segmentation for MR brain scan, emphazing the three regularization levels.

\section{Evaluation}

We implemented our approach in a simulated multiagent system where agents cooperate through a shared space memory (see Fig. 8). Based on various experiments we set the size of territories to $20 \times 20 \times 20$ voxels, resulting from 300 to 500 distributed agents. We set the parameters $\delta_{\text {keep }}=0.3$ and $\delta_{\text {replace }}=1.0$ for model correction and $M A X_{\text {tissue_estimation_restart }}=5$ to ensure the convergence of DILEM. The evaluation was performed using both phantoms and real $3 \mathrm{~T}$ brain scans. We used the normal BrainWeb phantoms database from the McConnell Brain Imaging Center [16]. These phantoms are generated from a realistic brain anatomical model and a MRI simulator that simulates MR acquisition physics, in which different values of nonuniformity and noise can be added. Because these 


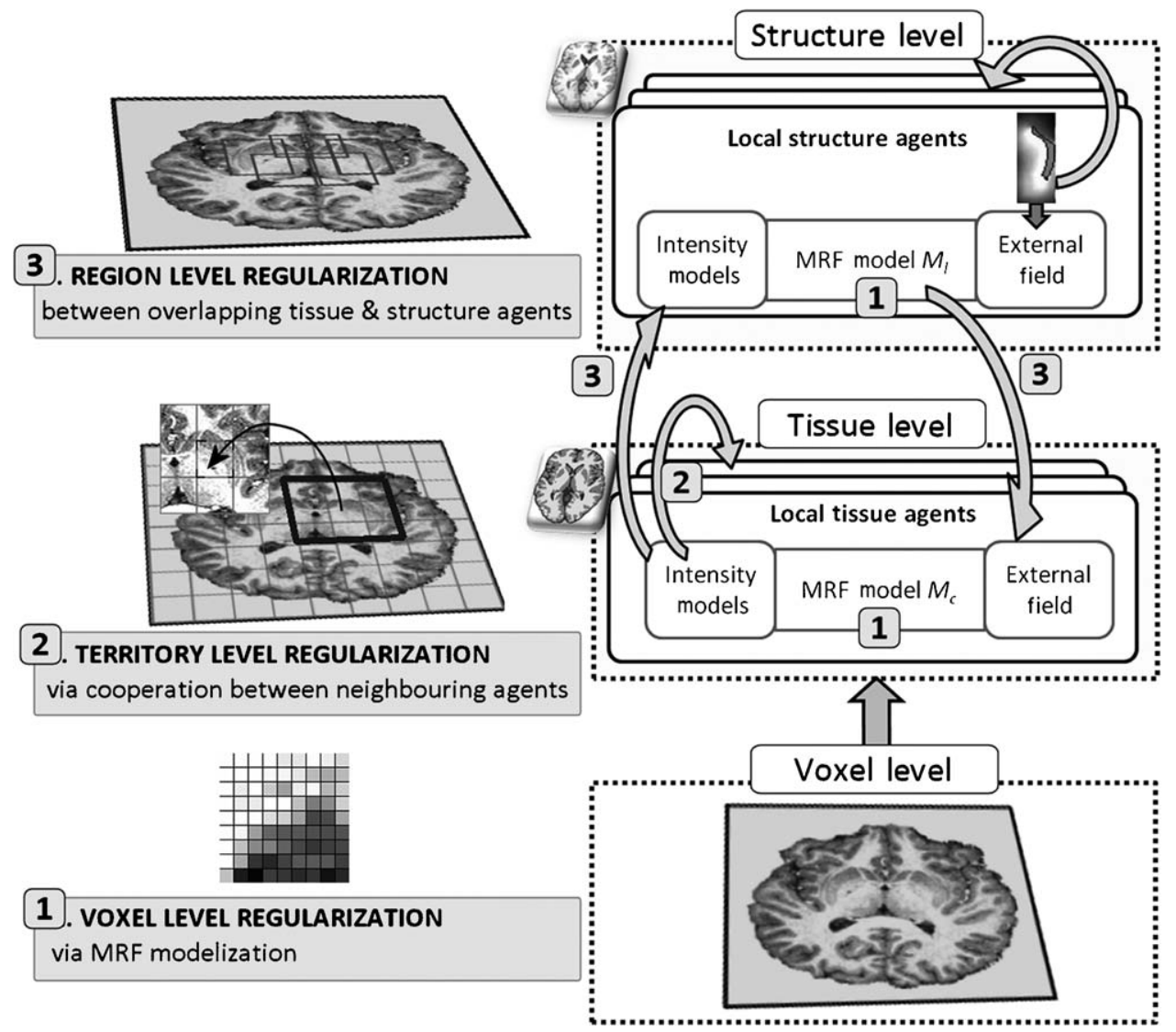

Figure 7 Synthetic view of the agent-based approach with the different regularization levels.

images are simulated we can quantitatively compare our tissue segmentation to the underlying tissue generative model to evaluate the segmentation performances. We performed this evaluation using the Jaccard similarity metric which measures the overlap between a segmentation result and the gold standard. By denoting by $\mathrm{TP}_{k}$ the number of true positives for class $k, \mathrm{FP}_{k}$ the number of false positives and $\mathrm{FN}_{k}$ the number of false negatives the Jaccard similarity metric is given by

$\mathcal{J}_{k}=\frac{\mathrm{TP}}{\mathrm{TP}+\mathrm{FN}+\mathrm{FP}}$
$\mathcal{J}_{k}$ takes its value in $[0,1]$ where the value 1 represents the complete agreement. We first evaluated the tissue segmentation performances provided by tissue agents only. We quantitatively compared our approach to two well known approaches, FSL [13] and SPM5 [2] (see Fig. 9). We ran FSL and SPM5 with default parameters similarly to several evaluations proposed in the literature. For LOCUS we did not optimize the parameters for the data used and kept these parameters both for BrainWeb phantoms and real $3 \mathrm{~T}$ brain scans. It shows comparable results and particularly more

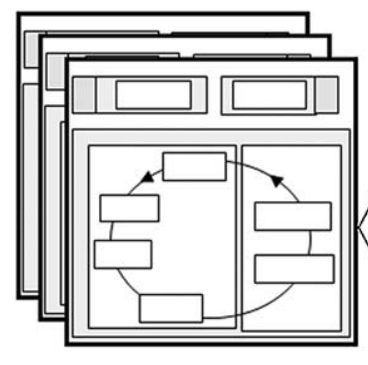

TISSUEAGENTS
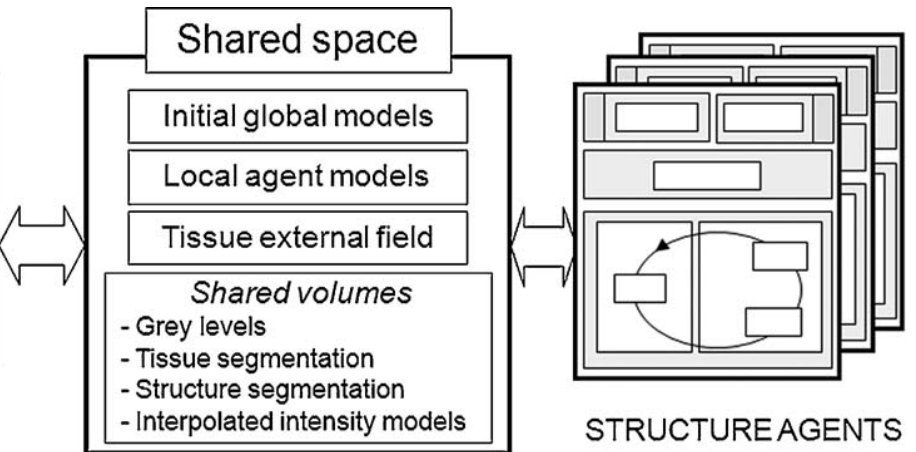

STRUCTUREAGENTS

Figure 8 Tissue and structure agents communicate via a shared space in the MA framework. 

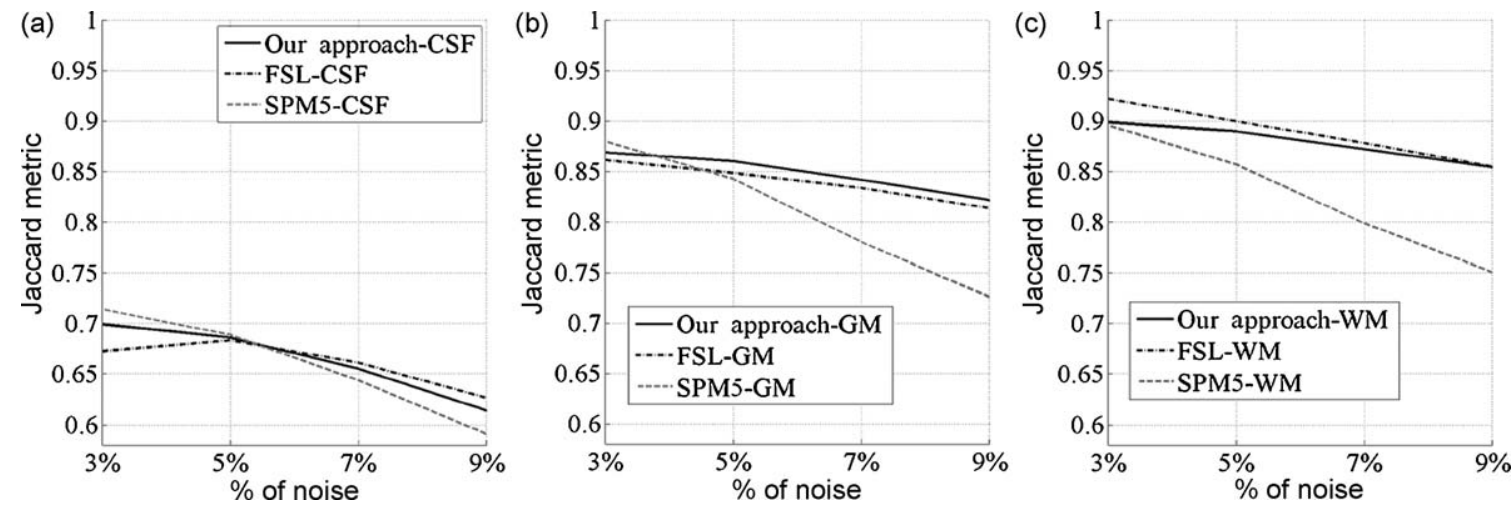

Figure 9 Comparison of our approach to FSL and SPM5 for tissue segmentation on the BrainWeb phantoms with $40 \%$ of non-uniformity and different noise values. Evaluation for class CSF (a), GM (b) and WM (c) classes.

robustness to noise than SPM5, whereas the computational time was approximately $4 \mathrm{~min}$ with our approach and, respectively $8 \mathrm{~min}$ and $14 \mathrm{~min}$ with FSL and SPM5 on a 4Ghz Pentium, 1Go RAM. Fig. 10 shows visual evaluation on a very high bias field brain scan. This image was acquired with a surface coil which provides a high sensitivity in a small region (here the occipital lobe). Such an acquisition is useful for functional imaging but results in highly non-uniform intensities. SPM5, which uses an a priori atlas, failed in the segmentation probably due to the difficulty to match the atlas with a surface coil brain acquisition. FSL did not estimate a correct bias field. Our local approach clearly appears to be more robust to very high intensity inhomogeneities. Fig. 11 shows results of cooperative tissue and structure segmentation. Tissue segmentation in the region of the putamens is clearly improved (Fig. 11C) by the regularization provided by structure segmentation agents. In addition to these competitive performances, our platform allows us to demonstrate some interesting properties of this local approach. We have developed a specific user interface to track the execution of each agent and to follow the segmentation process (see Fig. 12). Fig. 13 evaluates the segmentation on a high non-uniformity image for different size of agent territory. It shows that large territory sizes result in poor performance because the estimation is perturbed by non-uniformity. Smaller territory

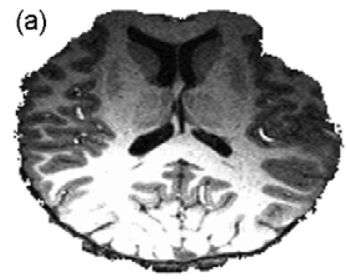

(b)

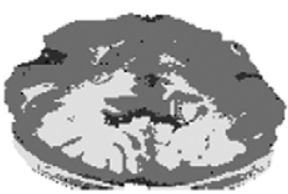

(c)

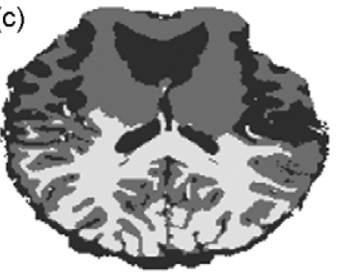

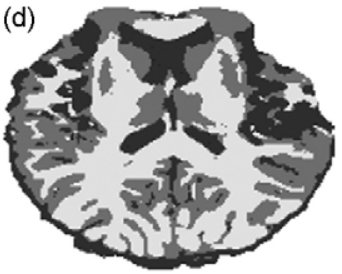

Figure 10 Evaluation on a MR brain scan with very high intensity non-unformity (a). Tissue segmentation provided by SPM5 (b), FSL (c) and our approach (d).
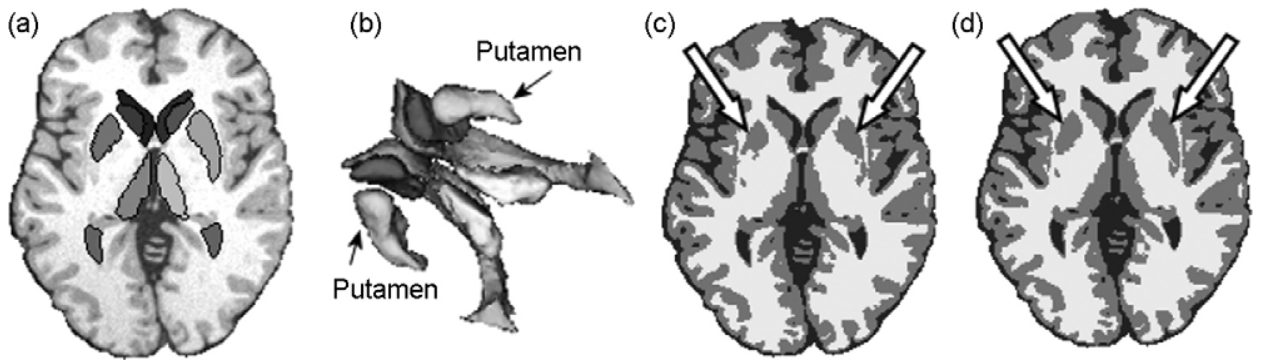

Figure 11 Cooperative tissue and structure segmentation: structure segmentation (a) with 3D rendering (b) shows good results with computational time less than $15 \mathrm{~min}$. Image (d) shows visual improvement in tissue segmentation compared to the segmentation produced by tissue agents only (c) (see putamens). 


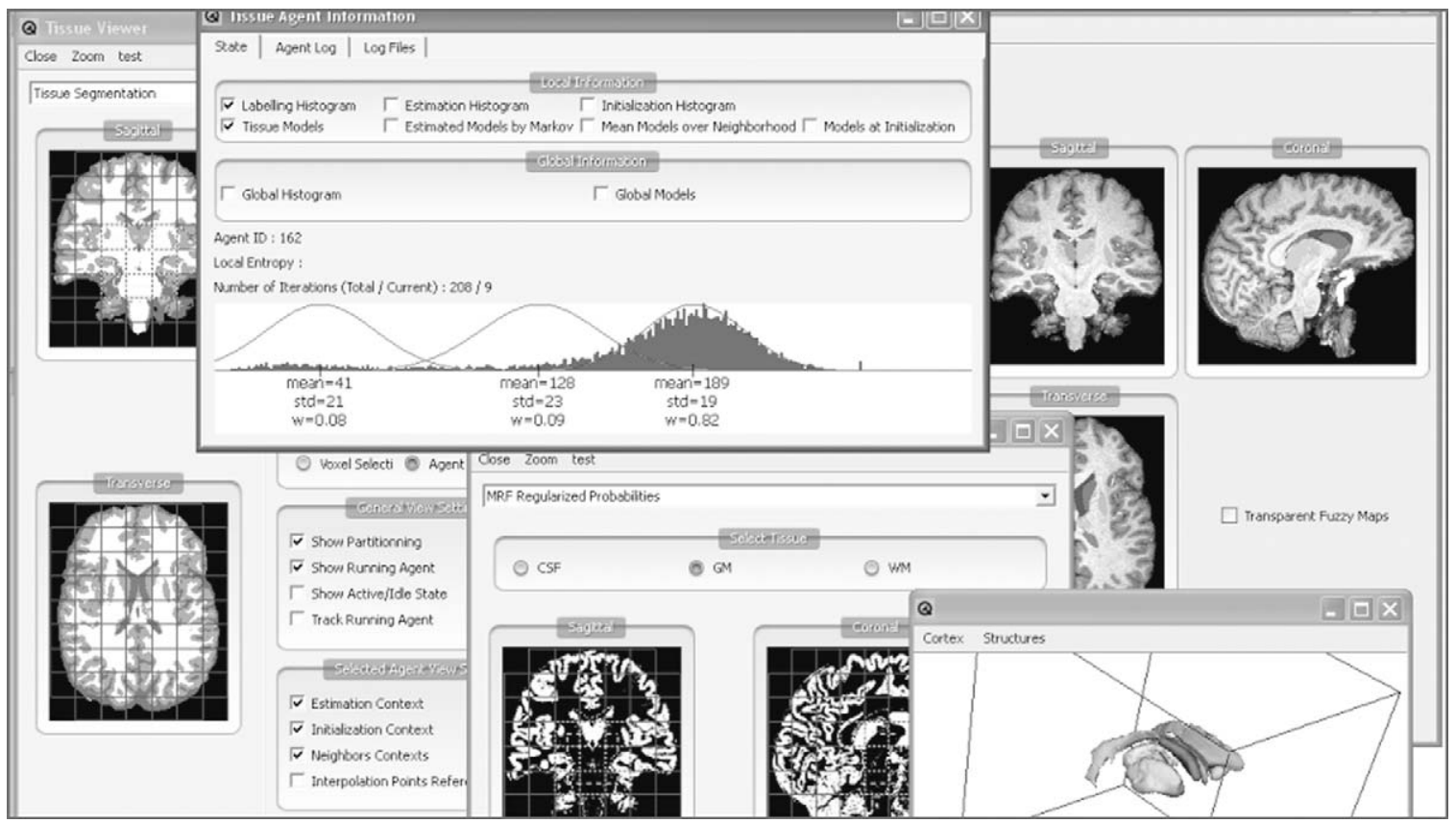

Figure 12 Graphical interface developed to follow the evolution of each agent during the segmentation.

sizes allow to better model local intensity distributions but need to be large enough to correctly estimate models. In practice, based on different experiments, territory size from $15 \times 15 \times 15$ voxels to $25 \times 25 \times 25$ voxels gives good results. Fig. 14 shows the number of DILEM iterations for some territories and illustrates the activity of four tissue agents immersed in the system. Agent 1 (see Fig. 14.c1) does not need model correction because all classes are sufficiently represented in its territory. The segmentation is then performed based only on local model estimation. Agent 2 (see Fig. 14.c2) has only a few brain voxels in its territory and then starts with unreliable knowledge. After initialization it waits for the neighboring agents to wake it up. Then only one model correction and a few DILEM iterations number are needed to segment its territory. It is likely that the local model is completely replaced by the neighouring mean model during the model correction. This model appear to be consistent with the few voxels of its territory and then DILEM converge very quickly. Some model checkings are performed during the $\mathcal{B}_{\text {LocalAgent_Stabilized }}$ behavior but do not activate the agent again. Agent 3 (see Fig. 14.c3) requires model corrections but requires a lot of DILEM iteration to get an agreement with its neighborhood. The local model and the mean model in neighborhood appear

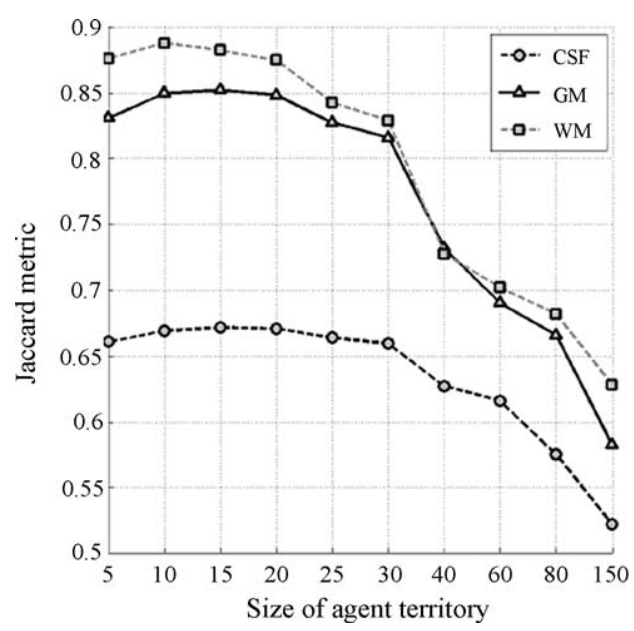

\begin{tabular}{|c|c|}
\hline \multicolumn{2}{|c|}{ Size of agent Number of } \\
\hline territory & agents \\
\hline 5 & 17422 \\
\hline 10 & 2474 \\
\hline 15 & 807 \\
\hline 20 & 379 \\
\hline 25 & 212 \\
\hline 30 & 137 \\
\hline 40 & 76 \\
\hline 60 & 18 \\
\hline 80 & 8 \\
\hline 150 & 1 \\
\hline
\end{tabular}

Figure 13 Influence of tissue agent territory size on the robustness to intensity non-uniformity, using the BrainWeb phantom with $3 \%$ of noise and $100 \%$ of non-uniformity. 
(a)

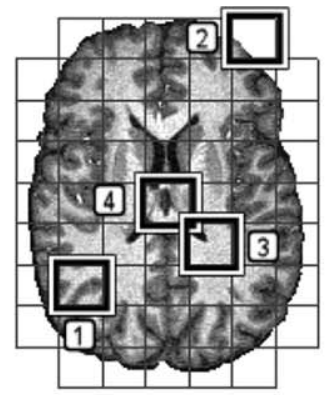

(b)

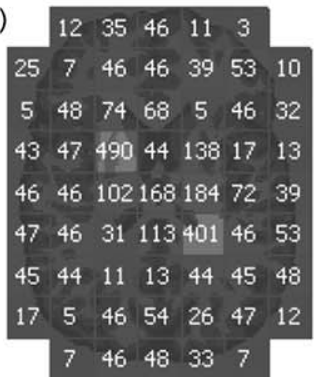

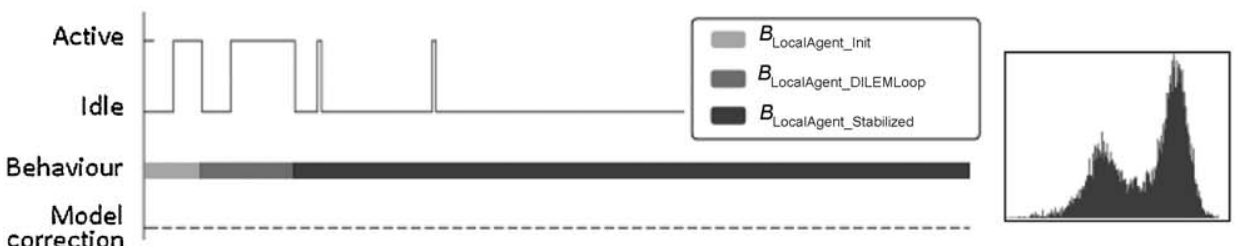

(c1)
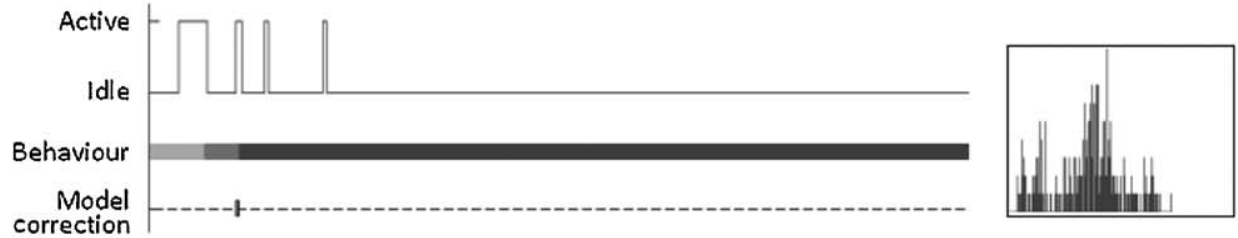

(c2)

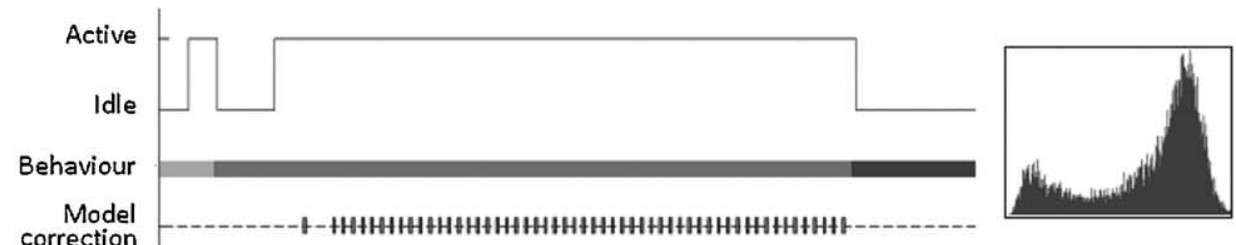

(c3)

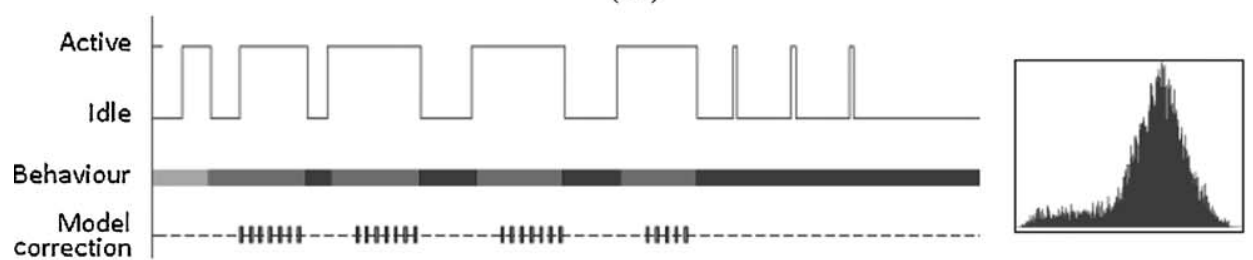

(c4)

Figure 14 Our implementation allows to observe the activity of each agent. (b) Shows the number of DILEM loop for each agent. (c1)-(c4) Show the execution chronograms for agents (1)-(4) pointed by (a) and the local histogram computed on the agent territory. It illustrates different behaviors for agents immersed in the system.

to be in competition to get the stabilization of the segmentation. Agent 4 (see Fig. 14.c4) requires model corrections as well. However, after a first agreement with the mean model in neighborhood (B $\mathcal{B}_{\text {LocalAgent_Stabilized }}$ behaviour) model checking restarts the estimation three times to get a final agreement. In Fig. 15(b) we computed for different agent positions the proportion of agents that require low or high DILEM iterations to reach the conver- gence. This graphic was computed from an average of eight BrainWeb segmentations for different values of noise (3\%, $5 \%, 7 \%$ and $9 \%$ ) and inhomogeneities $(20 \%$ and $40 \%)$. It shows that agents with a very few DILEM iterations are localized in a peripheral region of the brain. Agents with a large DILEM iterations number are mainly localized between 40 and $80 \mathrm{~mm}$, maybe because of the complexity to segment the grey matter of the cortex in this low 
(a)

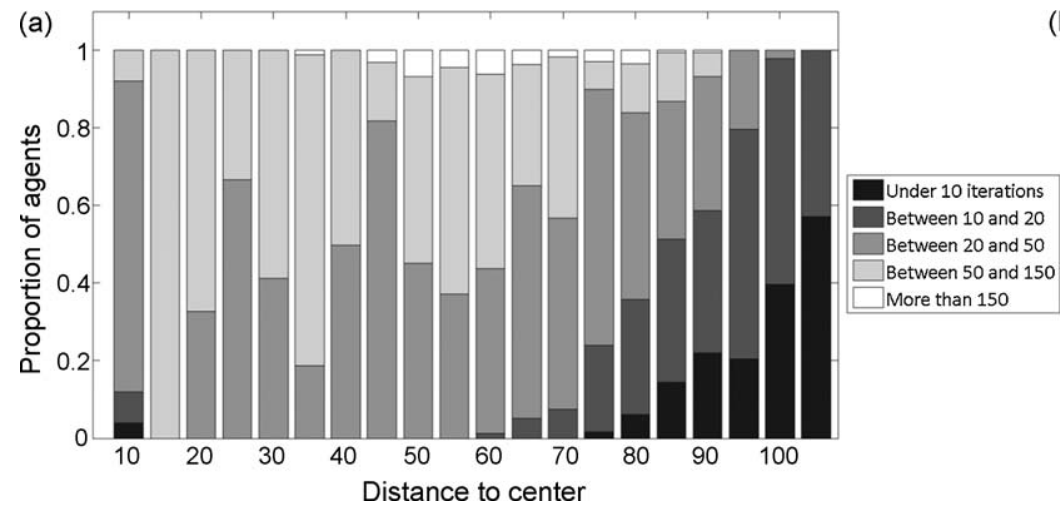

(b)

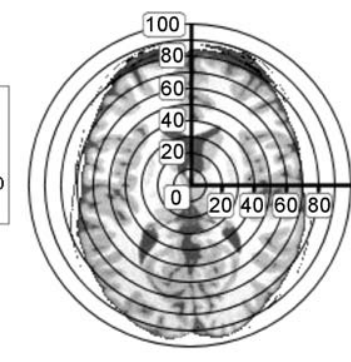

Figure 15 (a) Shows for various agent positions the proportion of agents that require high or low number of DILEM iterations to converge, computed from an average of eight BrainWeb segmentations. The position is defined by the distance from the agent territory center to the brain center (see (b)).

contrast region which contains a lot of partial volume voxels.

\section{Discussion and conclusion}

Embedding local EM procedures in a multiagent paradigm for the estimation of MRF parameters provides different interesting properties for image segmentation. First, local models accurately fit local image features. Second, local EM execution is adapted to local image complexity; more time is devoted to difficult-to-segment regions, lowering the global computational burden. Third, it allows a multilevel regularization. Such an approach requires the introduction of various cooperation and coordination mechanisms to ensure the global consistency of local models. Coordination is introduced to (1) ensure the spatial knowledge diffusion and (2) prevent agents from starting with poor quality knowledge. Cooperation provides different nature of regularization: regularization at a territory level with the DILEM algorithm to correct under-represented classes and regularization at a regional level to combine different kinds of knowledge. This approach is particularly suitable for MR brain scans segmentation. Accurate segmentation of MR brain scan must take into account both noise and intensity non-uniformity actifacts. The local perturbation produced by noise is handled by MRF. Intensity non-uniformity is a more global perturbation. It requires spatial adaptation of the models over the volume. Instead of estimating a spatial bias field model $[1,2]$ or using non-tractable non-stationary MRFs, our agent-based MRF approach compliments the local MRF regularization level with a global regularization level via the DILEM estimation procedure. In addition, we consider two levels of knowledge as regards to MR brain scans; tissue knowledge at a local level, and subcortical brain structure knowledge at a regional level. In general, these two levels are processed independently $[14,15]$. Rather, we consider that they are linked and must be used in a common setting. We show how to introduce a priori anatomical knowledge expressed by fuzzy spatial relations in the MRF framework to segment several subcortical structures. We then show how to take advantage of our agent-based Markovian framework to combine MRF models of tissues and structures. Subcortical structure segmentation then appears as an additional level of regularization; tissue and structure models are mutually constrained in their convergence, making them gradually more accurate and optimal. To our knowledge, distributed EM algorithms are seldom considered in the literature. Nowak [17] proposed a distributed EM algorithm for density estimation in sensor networks. Mensink et al. [18] proposed Multi-Observation Newscast EM for multi-camera tracking where every camera learns from both its own observations and communication with other cameras. These approaches are envisaged in a data fusion context: the algorithm is distributed but only one model is considered. No model correction between local models is performed.

Our implementation provides an interesting visualization tool which allows us to track specific agents and explore local knowledge such as local segmentation errors or local intensity models. The results are comparable to other algorithms with lower computational time. It appears to be robust to the intensity non-uniformity without any bias field assumption and estimation. Note that we currently consider a regular cubic partitioning for the DILEM algorithm but other methods like adaptive or spherical partitioning can be envisaged. This approach is currently extended to deal with patho- 
logical images encountered in the case of multiple sclerosis or stroke diseases. Finally, MRF agentbased computing appears as an interesting and modular tool for complex image segmentation.

\section{References}

[1] Wells WM, Grimson WEL, Kikinis R, Jolesz FA. Adaptative segmentation of MRI data. IEEE Transactions on Medical Imaging 1996;15(4):429-42.

[2] Ashburner J, Friston K. Unified segmentation. Neurolmage 2005;26:839-51.

[3] Germond L, Dojat M, Taylor C, Garbay C. A cooperative framework for segmentation of MRI brain scans. Artificial Intelligence in Medicine 2000;20:77-93.

[4] Richard N, Dojat M, Garbay C. Distributed Markovian segmentation: application to MR brain scans. Pattern Recognition 2007;40(12):3467-80.

[5] Geman S, Geman D. Stochastic relaxation, Gibbs distributions and the Bayesian restoration of images. IEEE Transactions on Pattern Analysis and Machine Intelligence 1984;6:721-41.

[6] Besag J. On the statistical analysis of dirty pictures. Journal of the Royal Statistical Society 1986;48:259-302.

[7] Celeux G, Forbes F, Peyrard N. EM procedures using mean field-like approximations for model-based image segmentation. Pattern Recognition 2003;36(1):131-44.

[8] Dempster AP, Laird NM, Rubin DB. Maximum likehood from incomplete data via EM algorithm. Journal of the Royal Statistical Society 1977;39:1-38.

[9] Scherrer B, Dojat M, Forbes F, Garbay C. MRF agent based segmentation: Application to MRI brain scans. In: Bellazzi R, Abu-Hanna A, Hunter J, editors. Proceedings of the 11th conference on artificial intelligence in medicine, AIME 2007. Artificial Intelligence in Medicine 2007;13-23.
[10] Scherrer B, Dojat M, Forbes F, Garbay C. LOCUS: LOcal Cooperative Unified Segmentation of MRI brain scans. In: Ayache N, Ourselin S, Maeder A, editors. Proceedings of the 10th international conference on medical image computing and computer assisted intervention, MICCAI2007. 2007. p. 219-27.

[11] Held K, Kopps ER, Krause BJ, Wells WM, Kikinis R, MullerGartner HW. Markov random field segmentation of brain MR images. IEEE Transactions on Medical Imaging 1997;16(6): 878-86.

[12] Van Leemput K, Maes F, Vandermeulen D, Suetens P. Automated model-based tissue classification of MR images of the brain. IEEE Transactions on Medical Imaging 1999;18(10): 897-908.

[13] Zhang Y, Brady M, Smith S. Segmentation of brain MR images through a hidden Markov random field model and the expectation-maximisation algorithm. IEEE Transactions on Medical Imaging 2001;20(1):45-7.

[14] Barra V, Boire J. Automatic segmentation of subcortical brain structures in MR images using information fusion. IEEE Transactions on Medical Imaging 2001;20(7):549-58.

[15] Colliot O, Camara O, Bloch I. Integration of fuzzy spatial relations in deformable models-application to brain MRI segmentation. Pattern Recognition 2006;39(8):140114

[16] Collins D, Zijdenbos A, Kollokian V, Sled J, Kabani N, Holmes $C$, et al. Design and construction of a realistic digital brain phantom. IEEE Transactions on Medical Imaging 1998;17(3): 463-8.

[17] Nowak R. Distributed EM algorithms for density estimation and clustering in sensor networks. IEEE Transactions on Signal Processing 2003;51:2245-53.

[18] Mensink T, Zajdel W, Krose B. Distributed EM learning for appearance based multi-camera tracking. In: Proceedings of the first ACM/IEEE international conference on distributed smart cameras, ICDSC'07; 2007. p. 178-85. 